\title{
Beobachtungen über die Chloroplastenteilung bei einigen Blütenpflanzen
}

\author{
Von \\ Seikan Kusunoki und Yoshio Kawasaki \\ Himeji-Kôtôgakkô, Japan
}

Eingegangen am 30 . Oktober 1936

Einleitung. Daß die Chloroplasten, sowohl bei Kryptogamen als auch bei Blütenpflanzen, durch Teilung sich vermehren, wurde zuerst von KIYoHaRA (1926) und etwas später von KASSMANN (1926) und GUILLIERMOND (1927) ${ }^{1)}$ durch Dauerbeobachtungen verfolgt. Nach den Angaben KIYOHARAs wird das letzte Stadium der Choloroplastenteilung, das uns interessiert, stets mitten in der Nacht beobachtet.

Dieser Befund scheint uns deshalb bemerkenswert $\mathrm{zu}$ sein, weil er uns nahelegt, unter welchen physikalisch-chemischen Bedingungen die betreffende Teilung der Chloroplasten bewirkt wird, mit anderen Worten, durch welchen Mechanismus der Teilungsvorgang der Chloroplasten vor sich geht.

Wenn wir zunächst bedenken, daß die im Tageslicht gebildeten Assimilate während der Nacht umgewandelt und zum Wachstum der Pflanze verbraucht werden, können wir uns leicht vorstellen, wie weit die physikalisch-chemischen Bedingungen in der Zelle beim Tageslicht von denen in der Nacht abweichen.

Wenn wir noch auf die wohlbekannte Tatsache hinweisen dürfen, daß die meisten Pflanzen in der Dunkelheit viel stärker als beim Sonnenlicht wachsen, können wir uns leicht davon überzeugen, daß unsere erste Aufgabe sein muß, den Befund KIYOHARAs zu bestätigen, wenn wir den Mechanismus der Chloroplastenteilung untersuchen wollen.

Im Nachstehenden wird über die Ergebnisse unserer diesbezüglichen Untersuchungen berichtet, welche wir in diesem Sommer im Botanischen Institut der Himeji-Kôtôgakkô ausgeführt haben.

Material und Methode. Als Untersuchungsmaterial wurden die Wasserpflanze Utricularia vulgaris L. und die Landpflanze Conandron ramondioides SIEB. et Zucc. verwendet, die in der Nähe von Himeji in Mitteljapan gesammelt wurden.

1) Ausführliche Literaturangaben über Chloroplastenteilung finden sich in einer Anzahl von Büchern, wie z.B. in : SHARP, Introduction to Cytology, 1934; SCHÜRHOFF, Die Plastiden, 1924 ; KiYohara, Zur Schimper-MeYerschen Theorie der Vermehrung der Chloroplasten 1935, u.a. 
Die Wahl der ersten Art geschah deshalb, weil die abgeschnittenen Stengel dieser Pflanze, wenn sie völlig unter Wasser gehalten werden, lange Zeit lebensfähig bleiben und die Wahl der zweiten, weil ein junger, vollständig von der Pflanze getrennter Sproß, dessen Wurzeln in Wasser gehalten werden, ebenfalls lange Zeit lebensfähig bleibt.

Es wurde nach der 1 schon von KIYOHARA angewandten Methode verfahren : Wie Abb. 1 u. 2 zeigen, wurden zur Beobachtung hohlgeschliffene Objektträger angewandt. Das basale Ende des für die Untersuchung bestimmten, abgetrennten Pflanzenteilchens wird dabei in den mit Wasser gefüllten Hohlraum getaucht. (Im Falle 1 befindet sich auch
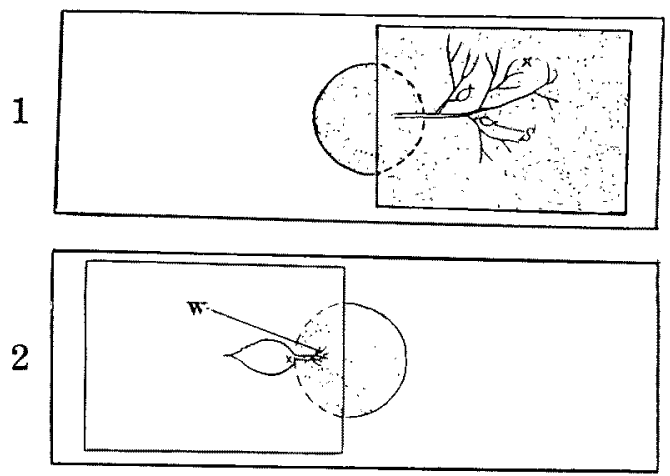

Abb. 1, 2. 1. Utricularia vulgaris. 2. Conandron ramondioides. $\times 4 / 5$. Hohlgeschliffener Objektträger mit Beobachtungsmaterial; die Punktierung bezeichnet den mit Wasser bedeckten Teil. $\times$ beobachtete Stelle, s Bläschen, $w$ Wurzel. unter dem Deckglas Wasser, im Falle 2 nicht.) Auf diese Weise kann man die Vorgänge in den Zellen ungestört beobachten.

Beobachtungen. In den Zellen jüngerer Stengel von Utricularia vulgaris $\mathrm{L}$. finden sich zahlreiche hellgrüne, ellipsenförmige Chloroplasten, deren Gestalt meistens bald kokon- oder hantelförmig wird. In den Zellen ausgewachsener Stengel jedoch sind Chloroplasten von den genannten Formen sehr selten — fast alle haben die Gestalt grüner Kugeln.

Bei Utricularia ist eine Bewegung der Chloroplasten infolge von Protoplasmaströmung nicht zu beobachten. Vielmehr konnte festgestellt werden, daß sich der ganze Teilungsvorgang eines Chloroplasten auf ein- und derselben Stelle abspielt und zwar in folgender Weise:

Die anfänglich rundliche Gestalt der Chloroplasten wird allmählich kokonförmig und dann beginnt der Teilungsvorgang: Durch Bildung einer Furche beginnt die Gestalt sich zur Hantelform zu verändern, bis sich durch weiter und weiter fortschreitende Vertiefung der Furche schlieblich die Teilung vollzieht.

Dieser Vorgang stimmt mit den Angaben KIYoHaras vollständig überein (Abb. 3). Merkwürdig ist dabei, daß bei einer Hälfte der hantelförmigen Chloroplasten manchmal eine zweite Furchung derart einsetzt, daß im ganzen schließlich drei Teilgebilde entstehen (Abb. $3 \mathrm{~d})$. Während bei einer Reihe von Chloroplasten derselben 

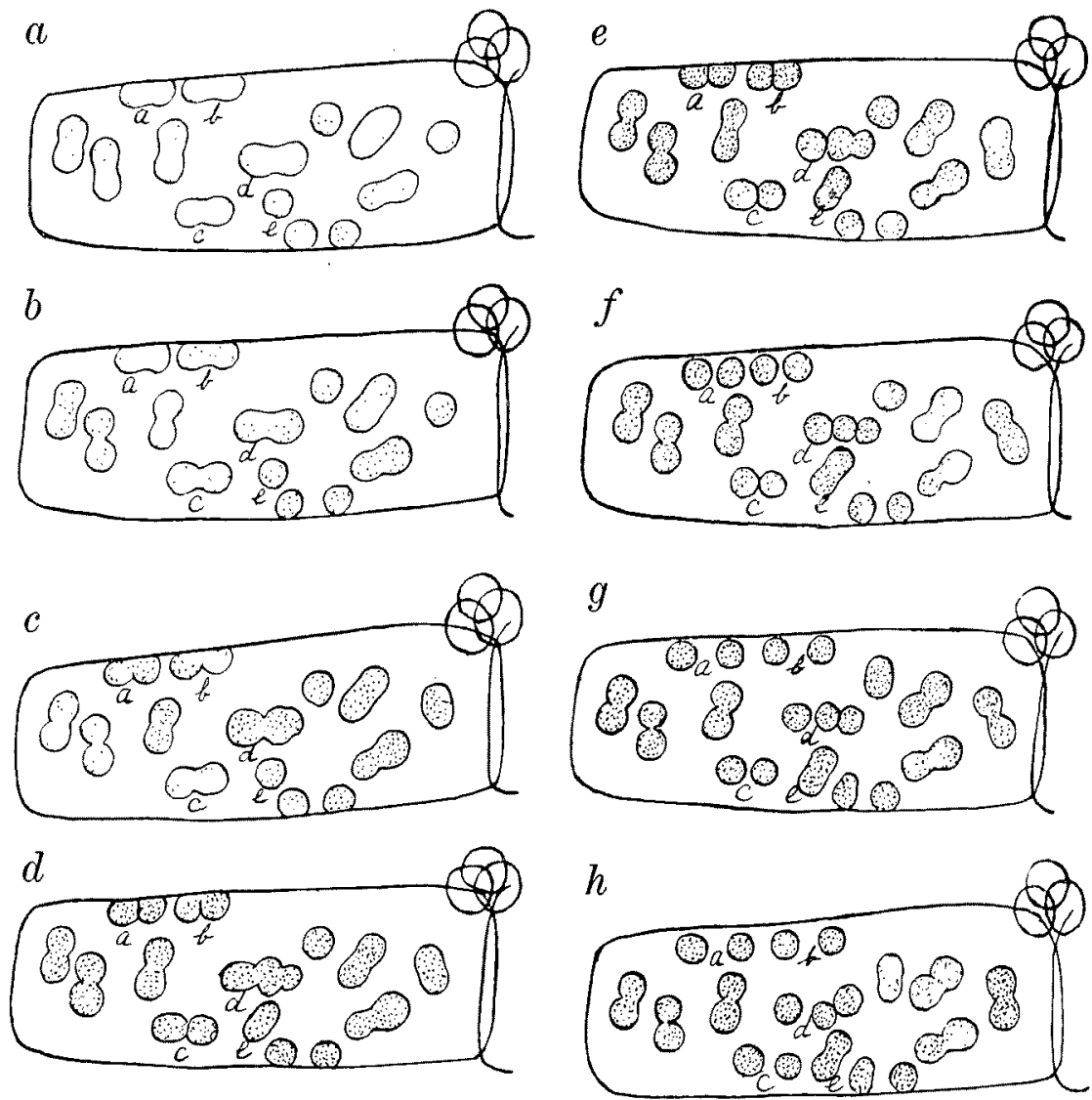

Abb. 3. Teilungsvorgang der Chloroplasten bei Dauerbeobachtung :
(a) 9 Uhr abends,
(b) 10.30 abends,
(c) 11 Uhr abends, (d) 11.30 abends,
(e) 12.30 morgens,
(f) 1.30 morgens,
(g) 2.30 morgens,
(h) 3.30 morgens.

Zelle die Trennung sich binnen einer Nacht gänzlich vollzog, war sie bei anderen Chloroplasten nur bis zur Hantelform fortgeschritten. Zwar war die Beobachtung des Teilungsvorganges so schwierig, daß sie nicht auf eine zweite Nacht ausgedehnt werden konnte, aber mit Sicherheit kann gesagt werden, daß sie sich nicht am Tage vollendet. Es sei noch erwähnt, daß unter 14 Chloroplasten in einer Zelle nur bei 4 die Beobachtung des Teilungsvorganges bis zur Vollendung möglich war.

Conandron ramondioides SIEB. et ZuCC. In der Mitte der sechseckigen Zellen konnten wir immer je ein sternförmiges Aggregat von Kalziumoxalatkristallen beobachten. Die ausgewachsenen Zellen aber waren mit dunkelgrünen rundlichen Chloroplasten erfüllt. In solchen ausgewachsenen Zellen war keine Teilung der Chloroplasten mehr zu beobachten. In jungen Zellen, in welchen noch Chloro- 
plastenteilung vor sich geht, ist die Zahl der Chloroplasten viel geringer, als in alten, worüber KIYOHARA wie folgt berichtet hat:

„.... Und hat die Gesamtzahl der Chloroplasten, die in einer Zelle enthalten sind, eine bestimmte Höhe erreicht, d.h. hat eine Zelle ihre Entwicklungsfähigkeit eingebüßt, so verlieren auch die Chloroplasten ihre Teilbarkeit und erhalten ihre eigentliche Gestalt."

An normalen Fällen läßt sich diese Beobachtung nur bestätigen. Die Chloroplasten von rundlicher Gestalt werden wiederum zunächst kokon- und dann hantelförmig, aber dieser Prozeß vollzieht sich im allgemeinen untertags und dauert bis zum Abend, die Teilung aber geht nach unseren Beobachtungen wie bei Utricularia vulgaris nur in der Nacht vor sich. Die Chloroplasten dieser Pflanze werden durch die Protoplasmaströmung mitbewegt, was unter dem Mikroskop ganz deutlich festgestellt werden kann. Die Geschwindigkeit der sich bewegenden Chloroplasten ist aus folgenden Zahlen zu ersehen:

binnen $13 / 4$. Stunden (von 8.20 bis 10.05 ) abends: $13.5 \mu$ binnen $11 / 2$ Stunden (von 9.10 bis 10.40) morgens: $16.2 \mu$

Die Chloroplasten bewegen sich dabei, einer hinter dem anderen, von der Zellwand her in einem Bogen gegen das Kristallaggregat in der Mitte der Zelle hin. Während dieser Zeit schreitet die Teilung bis zur Vollendung fort. Die Länge der während der Teilung der Chloroplasten zurückgelegten Bahn beträgt nur $1 / 3$ bis $1 / 5$ des
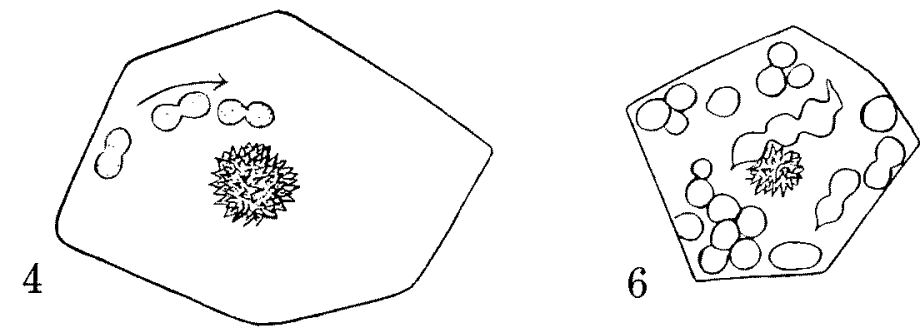

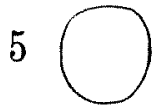

$a$

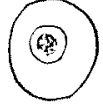

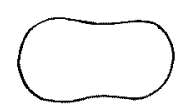

$b$

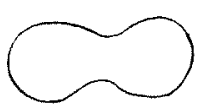

$c$

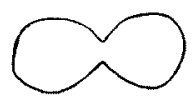

$d$

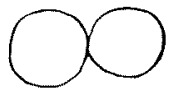

$e$
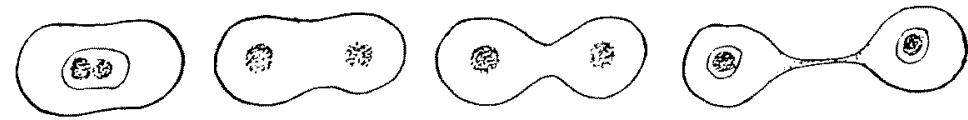

Abb. 4-7. Conandron ramondioides. 4. Eine Zelle. 5. Teilungsvorgang der Chloroplasten bei Dauerbeobachtung. a. 29. Juni 9.20 a.m.; b. 30 . Juni 8.20 p.m.; c. 30. Juni 10.30 p.m.; d. 1. Juli 12.45 a.m.; $e$. 1. Juli 1.20 a.m. 6. Eine Zelle, in der ein dreiteiliger Chloroplast sichtbar ist. 7. Mit Alkohol behandelte Chloroplasten. 
Gesamtumfanges der Zelle (Abb. 4). Der zeitliche Verlauf des Teilungsvorganges ist aus Abb. 5 ersichtlich.

An dieser Stelle möchten wir nochmals auf KIYoHARAs Beobachtung hinweisen, daß die Chloroplasten ausgewachsener Zellen ihr Teilungsvermögen verloren haben. Durch geringe Veränderung der Bedingungen können jedoch nach unseren Beobachtungen die Chloroplasten auch solcher Zellen zur Teilung veranlaßt werden. Beide Arten der Teilung, normale und anormale konnten dadurch hervorgerufen werden, daß das Blatt, an dessen Zellen die Beobachtungen vorgenommen wurden, in Wasser eingetaucht wurde. In diesem Falle drängten sich die Chloroplasten, die bis dahin in der ganzen Zelle homogen zerstreut lagen, zu einzelnen Gruppen zusammen, sodaß dazwischen größere Zwischenräume entstanden. Die noch in diesen Räumen zerstreut befindlichen Chloroplasten vermochten sich wieder zu teilen (Abb. 6).

Es ist bemerkenswert, daß solche Veränderungen nur durch Wasser hervorgerufen werden konnten.

Bei der Chloroplastenteilung in frischem Material dieser Pflanze wurde ein langausgezogener Stromastrang nicht beobachtet, wie er von KIYOHARA als bemerkenswert genannt wird. Wurde das Material aber in Alkohol getaucht, so wurde eine solche Brücke deutlich erkennbar (Abb. 7).

Durch diese Untersuchung wird mit Sicherheit bestätigt, daß das letzte Stadium der Chloroplastenteilung, wie es von KIYOHARA bei Hydrilla verticillata festgestellt wurde, stets mitten in der Nacht beobachtet wird. Und zugleich konnten wir zwei neue Beispiele dafür ausfindig machen. 